\title{
A Linear-Time Algorithm for Computing the Diameters of the Incomplete WK-Recursive Networks
}

\author{
Min-Yang Su and Gen-Huey Chen \\ Department of Computer Science and Information Engineering, \\ National Taiwan University, Taipei, TAIWAN
}

\author{
Dyi-Rong Duh \\ Department of Electronic Engineering, \\ Hwa Shia Junior College, Taipei, TAIWAN
}

\begin{abstract}
The WK-recursive networks, which were originally proposed by Vecchia and Sanges, have suffered from the rigorous restriction on the number of nodes. Like the other incomplete networks, the incomplete WK-recursive networks have been proposed to relieve this restriction. In this paper, it is first shown that the structures of the incomplete WK-recursive networks are conveniently represented with multistage graphs. This representation can provide a uniform look at the incomplete WKrecursive networks. By its aid, a linear-time algorithm using the prune-and-search technique is presented for computing the diameters of the incomplete WK-recursive networks.
\end{abstract}

\section{Introduction}

One important step on designing a large multiprocessor system is to determine the topology of the interconnection network (network for short). In the recent decade, a number of networks have been proposed in the literature $[1,3,4,17,19,20,24]$. Among them, the WK-recursive networks [24] own two structural advantages: expansibility and equal degree. A network is expansible if no changes to node configuration and link connection are necessary when it is expanded, and of equal degree if all its node have the same degree no matter what the size is. A network with these two properties will gain the advantages of easy implementation and low cost when it is manufactured.

The WK-recursive networks, which were proposed by Vecchia and Sanges [24], represent a class of recursively scalable networks. They offer high degree of regularity, scalability and symmetry which very well conform to a modular design and implementation of distributed systems involving a large number of computing elements. A VLSI implementation of a 16-node WK-recursive network has been realized at the Hybrid Computing Research Center [25]. In this implementation, each node was implemented with the INMOS IMS T414 transputer, and the network processes were coded in Occam programming language. Later this prototype network was further extended to 64 nodes [25]. Recently two variants of the WK-recursive networks have been proposed in $[7,8]$.

Although the WK-recursive networks own many nice properties $[2,5,6,9-11,12,24,25]$, there is a rigorous restriction on the number of their nodes. As will become clear in the next section, the number of nodes in a WKrecursive network must satisfy $d^{t}$, where $d>1$ is the size of the basic building block and $t \geq 1$ is the level of expansion. Thus, as $d=4$, extra $3 \cdot 4^{7}=49152$ nodes are required to expand from a 7 -level WK-recursive network to an 8-level WK-recursive network. All of the networks mentioned above suffer from the same restriction. To relieve this restriction, some incomplete networks such as incomplete hypercubes [13], incomplete star networks $[14,18]$, incomplete rotator graphs [16], were proposed. Recently, the incomplete WK-recursive networks have been proposed by the authors [21]. From a practical point of view, the number of nodes is considered a multiple of $d$, where $d$ is the size of the basic building block. Thus, an incomplete WK-recursive network can be expanded or contracted in units of basic building blocks.

The distance between two nodes of a network is the smallest number of links that are contained in any path between them. The diameter of a network is the maximum distance between any pair of nodes. Since the diameter is usually considered the maximum transmission delay within a network, it is one of the most fundamental topological properties for the network. The diameters for the incomplete star networks and the incomplete rotator graphs are still unknown, although the diameters for the star networks [1] and the rotator graphs [3] have been obtained. Ravikumar et. al. [18] suggested an upper bound on the diameters of the incomplete star networks. For a special class of incomplete star networks that comprise $c k$-stars, where $1<c \leq k$, the diameters have been computed $[14,18]$. In this paper, using the prune-andsearch technique [15], an $O(t)$ time algorithm is proposed 
for computing the diameters of the incomplete WKrecursive networks, where $t$ is the level of expansion.

It will be shown in the next section that the structures of the incomplete WK-recursive networks can be conveniently represented with multistage graphs. In this way, the diameter problem on the incomplete WKrecurisve networks is reduced to the diameter problem on the multistage graphs. In the next section, the incomplete WK-recursive networks are formally defined, and their multistage graph representations are also introduced. In Section 3, a tight upper bound on the diameters is suggested. In Section 4, a linear-time algorithm for computing the diameters is proposed. Finally, this paper is concluded with some remarks in Section 5.

\section{Incomplete WK-Recursive Networks and Their Multistage Graph Representations}

In this section we first review the structures of the WK-recursive networks. The incomplete WK-recursive networks are then defined as the induced subgraphs of the WK-recursive networks. It has been shown convenient (see [21]) to express the structure of an incomplete WKrecursive network as a multistage graph. The multistage graph representation is also reviewed in this section.

The WK-recursive networks can be constructed recursively by grouping basic building blocks. Any complete graph can serve as a basic building block. For convenience, let $\mathrm{K}(d, t)$ denote a WK-recursive network of level $t$ whose basic building blocks are each a $d$-node complete graph, where $d>1$ and $t \geq 1 . K(d, 1)$, which is the basic building block, is a $d$-node complete graph, and $\mathrm{K}(d$, $t)$ for $t \geq 2$ is composed of $d \mathrm{~K}(d, t-1)$ 's that are connected as a complete graph. Each node of $\mathrm{K}(d, t)$ is associated with a $t$-digit identifier. The following definition is due to Chen and Duh [2].

Definition 2.1. The node set of $\mathrm{K}(d, t)$ is denoted by $\left\{a_{t-1} a_{t-2} \ldots a_{1} a_{0} \mid a_{i} \in\{0,1, \ldots, d-1\}\right.$ for $\left.0 \leq i \leq t-1\right\}$. Node adjacency is defined as follows: $a_{t-1} a_{t-2} \ldots a_{1} a_{0}$ is adjacent to (1) $a_{t-1} a_{t-2} \ldots a_{1} b$, where $0 \leq b \leq d-1$ and $b \neq a_{0}$, and (2) $a_{t-1} a_{t-2} \cdots$ $a_{j+1} a_{j-1}\left(a_{j}\right)^{j}$ if $a_{j} \neq a_{j-1}$ and $a_{j-1}=a_{j-2}=\ldots=a_{0}$, where $1 \leq j \leq t-1$ and $\left(a_{j}\right)^{j}$ represents $j$ conecutive $a_{j}$ 's. The links of (1), labeled 0 , are called substituting links. The links of (2), labeled $j$, are called $j$-flipping links (or simply flipping links). Besides, there are open links whose one end node is $(a)^{2}$, where $0 \leq a \leq d-1$, and the other end node is unspecified. The open links are labeled $t$.

Since each node is incident with $d$-1 substituting links and one flipping link (or open link), $\mathrm{K}(d, t)$ has
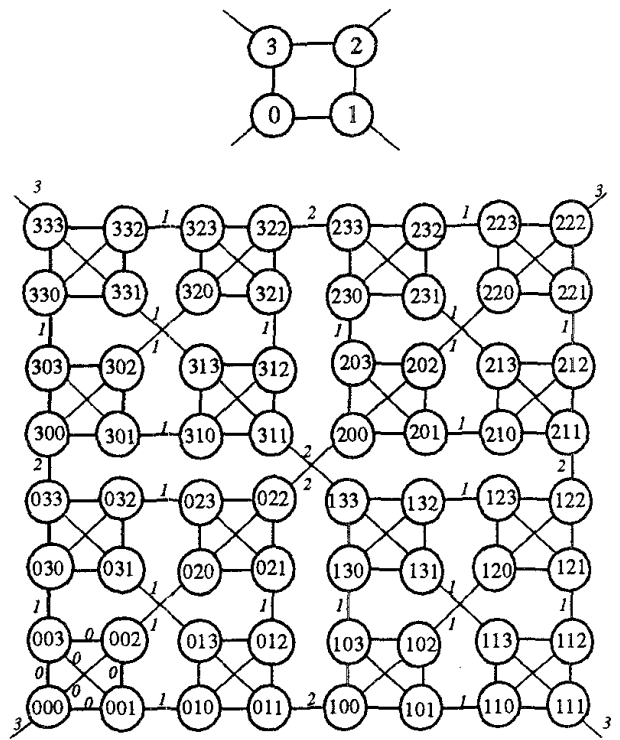

Figure 1. The structures of $K(4,1)$ and $K(4,3)$.

degree $d$. The structures of $K(4,1)$ and $K(4,3)$ are illustrated in Figure 1. Intuitively, the substituting links are those within basic building blocks, the $j$-flipping links each connect two embedded $\mathrm{K}(d, j)$ 's, and the open links are left for future expansion. For example, let us consider the incident links of node 311 in Figure 1. The one to node 133 is a 2 -flipping link, and the others are substituting links. Since the WK-recursive networks are recursive, $\mathrm{K}(d, t)$ contains $\mathrm{K}(d, i)$ 's, $1 \leq i<t$, as subgraphs (subgraph and subnetwork are used interchangeably in this paper). In the following we define these subgraphs formally.

Definition 2.2. Define $c_{t-1} c_{t-2} \ldots c_{r} \cdot \mathrm{K}(d, r)$ to be the subgraph of $\mathrm{K}(d, t)$ induced by $\left\{c_{t-1} c_{t-2} \ldots c_{r} a_{r-1} \ldots a_{1} a_{0} \mid a_{i} \in\right.$ $\{0,1, \ldots, d-1\}$ for $0 \leq i \leq r-1\}$, where $1 \leq r \leq t-1$ and $c_{t-1}, c_{t-2}$, $\ldots, c_{r}$ are all integers from $\{0,1, \ldots, d-1\}$.

For example, refer to Figure 1 where $31 \cdot \mathrm{K}(4,1)$ is the subgraph of $K(4,3)$ induced by $\{310,311,312,313\}$.

Definition 2.3. Node $a_{t-1} a_{t-2} \ldots a_{1} a_{0}$ is a $k$-frontier if $a_{k-1}=\ldots=a_{1}=a_{0}$, where $1 \leq k \leq t$.

Note that by Definition 2.3, a $k$-frontier is automatically an $l$-frontier for $1 \leq k<$. Both end nodes of a $k$-flipping link are $k$-frontiers. An embedded $\mathrm{K}(d, r)$, where $1 \leq r \leq t-1$, contains one $(r+1)$-frontier and $d-1 r$ frontiers. These $d$ frontiers are $2^{r}-1$ distant from each other. 
If we number the nodes of $\mathrm{K}(d, t)$ according to their lexicographical order, then an $N$-node incomplete WKrecursive network is the subgraph of $\mathrm{K}(d, t)$ induced by the first $N$ nodes. Throughout this paper, we use $\operatorname{IK}(d, t)$ to denote an $N$-node incomplete WK-recursive network, where $d^{t-1}<N<d^{t}$ and $N$ is a multiple of $d$.

The coefficient vector associated with an $N$-node $\operatorname{IK}(d, t)$ is a $(t-1)$-tuple using $d$-ary numbers $\left(b_{t-1}, b_{t-2}, \ldots\right.$, $b_{1}$ ) satisfying $N=b_{t-1} d^{t-1}+b_{t-2} d^{t-2}+\ldots+b_{1} d$, where $d^{t-1}<N<$ $d^{t}$ and $N$ is a multiple of $d . \operatorname{IK}(d, t)$ with coefficient vector $\left(b_{t-1}, b_{t-2}, \ldots, b_{1}\right)$ contains $b_{t-1} \mathrm{~K}(d, t-1)$ 's, $b_{t-2} \mathrm{~K}(d, t-2)$ 's, $\ldots$, and $b_{1} \mathrm{~K}(d, 1)$ 's. For $1 \leq m \leq t-1$, the $b_{m} \mathrm{~K}(d, m)$ 's are $b_{t-1} b_{t-2} \ldots b_{m+1} 0 \cdot \mathrm{K}(d, m), b_{t-1} b_{t-2} \ldots b_{m+1} 1 \cdot \mathrm{K}(d, m), \ldots$, and $b_{t-1} b_{t-2} \ldots b_{m+1}\left(b_{m}-1\right) \cdot \mathrm{K}(d, m)$. For example, $\operatorname{IK}(5,6)$ with coefficient vector $(2,3,0,4,0)$ contains the following subnetworks.

$0 \cdot \mathbf{K}(5,5), 1 \cdot \mathbf{K}(5,5)$

$20 \cdot \mathrm{K}(5,4), 21 \cdot \mathrm{K}(5,4), 22 \cdot \mathrm{K}(5,4)$

$2300 \cdot \mathrm{K}(5,2), 2301 \cdot \mathrm{K}(5,2), 2302 \cdot \mathrm{K}(5,2) 2303 \cdot \mathrm{K}(5,2)$

See Figure 2 where the structure of $\operatorname{IK}(4,3)$ with coefficient vector $(3,2)$ is illustrated. In the rest of this paper, a coefficient vector $\left(b_{t-1}, b_{t-2}, \ldots, b_{1}\right)$ is written as $\left(b_{t-1}, b_{t-2}, \ldots, b_{i}, *\right)$, provided $b_{i} \neq 0$ and $b_{i-1}=b_{i \cdot 2}=\ldots=b_{1}=$ 0 . For example, $(2,3,0,4,0)$ is written as $(2,3,0,4$, $*)$, and $(2,3,4)$ is written as $(2,3,4, *)$.

Let $G_{m}$ represent the subgraph of $\operatorname{IK}(d, t)$ with coefficient vector $\left(b_{t-1}, b_{t-2}, \ldots, b_{1}\right)$ induced by $b_{t-1} b_{t-2} \ldots$ $b_{m+1} 0 \cdot \mathrm{K}(d, m), b_{t-1} b_{t-2} \ldots b_{m+1} 1 \cdot \mathrm{K}(d, m), \ldots, b_{t-1} b_{t-2} \ldots b_{m+1}$ $\left(b_{m}-1\right) \cdot \mathrm{K}(d, m)$, where $1 \leq m \leq t-1$. For example, the $\mathrm{IK}(5$, 6) with $(2,3,0,4, *)$ mentioned above has $G_{5}$ containing $0 \cdot \mathrm{K}(5,5)$ and $1 \cdot \mathrm{K}(5,5), G_{4}$ containing $20 \cdot \mathrm{K}(5,4)$, $21 \cdot \mathrm{K}(5,4)$, and $22 \cdot \mathrm{K}(5,4), G_{3}$ empty, $G_{2}$ containing $2300 \cdot \mathrm{K}(5,2), 2301 \cdot \mathrm{K}(5,2), 2302 \cdot \mathrm{K}(5,2)$, and $2303 \cdot \mathrm{K}(5$, 2). For easy reference, we refer to $b_{t-1} b_{t-2} \ldots b_{m+1} j \cdot \mathrm{K}(d, m)$ as the $(j+1)$ th $K(d, m)$ of $G_{m}$, where $0 \leq j \leq b_{m^{-}}-1$.

According to the discussion above, the structure of $\operatorname{IK}(d, t)$ with coefficient vector $\left(b_{t-1}, b_{t-2}, \ldots, b_{i}, *\right)$ can be expressed as a multistage graph, denoted by $G_{t-1}+G_{t-2}+\ldots$ $+G_{i}$. There are $t-i$ stages, i.e., $G_{t-1}, G_{t-2}, \ldots$, and $G_{i}$. For example, the multistage graph representations of $\operatorname{IK}(5,6)$ with coefficient vector $(2,3,0,4, *)$ and $\operatorname{IK}(6,10)$ with coefficient vector $(4,2,4,3,4,1,1,3,1, *)$ are shown in Figure 3, where each $\mathrm{K}(d, m)$ within $G_{m}$ is drawn as a circle and the links within $G_{m}$ are omitted for conciseness. The links between $G_{m}$ and $G_{n}$, where $t-1 \geq m>n \geq i$, are $m$ flipping links, and they are called jumping m-flipping links if $m-n>1$. There are $\min \left\{b_{m}, b_{m-1}\right\} m$-flipping links connecting the $j$ th $\mathrm{K}(d, m)$ of $G_{m}$ and the $j$ th $\mathrm{K}(d, m-1)$ of $G_{m-1}$, where $1 \leq j \leq \min \left\{b_{m}, b_{m-1}\right\}$. Besides, there may exist some jumping flipping links and they can be determined according to the following theorem.

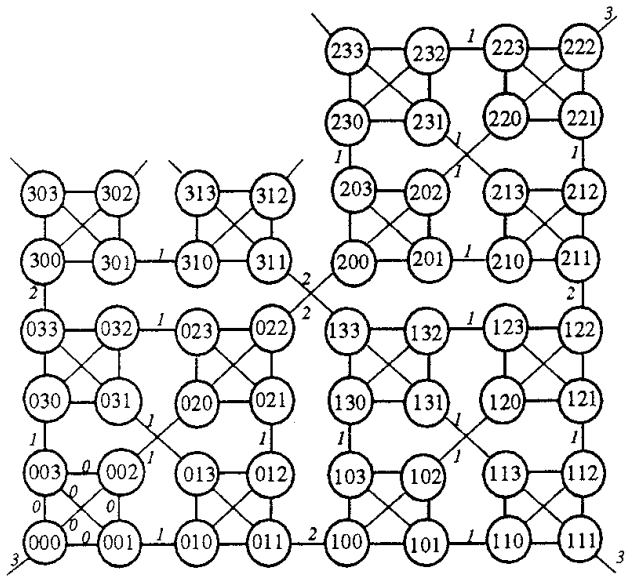

Figure 2 . The structures of $\operatorname{IK}(4,3)$ with coefficient vector $(3,2, *)$.

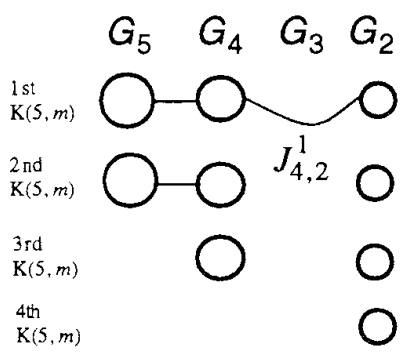

(a)

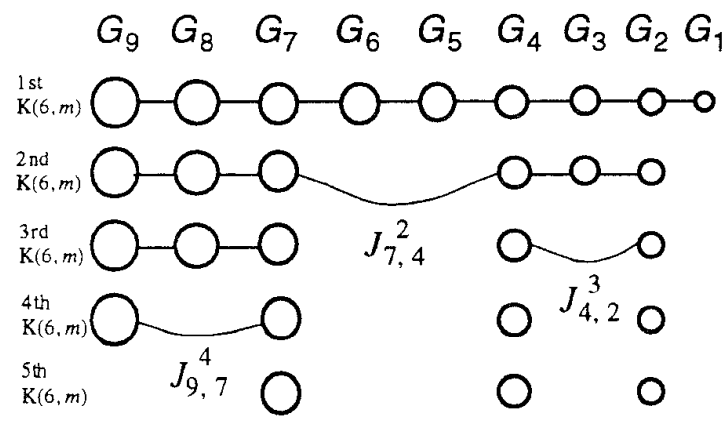

(b)

Figure 3. (a) $\operatorname{IK}(5,6)$ with ooefficient vector $(2,3,0,4, *)$ and (b) $\operatorname{IK}(6,10)$ with coefficient vector $(4,3,5,1,1,5,2,5,1, *)$.

Theorem 2.1.[22] For $\operatorname{IK}(d, t)$ with coefficient vector $\left(b_{t-1}, b_{t-2}, \ldots, b_{i}, *\right)$, one jumping $m$-flipping link exists between $G_{m}$ and $G_{n}$ if and only if $b_{m}>b_{m-1}=b_{m-2}=$ $\ldots=b_{n+1}<b_{n}$, where $t-1 \geq m>n \geq i$ and $m-n>1$. Moreover,

(1) this link connects the $(r+1)$ th $\mathrm{K}(d, m)$ and the $(r+1)$ th $\mathrm{K}(d, n)$, where $r=b_{m-1}=b_{m-2}=\ldots=b_{n+1}$, and 
(2) no other link exists between $G_{x}$ and $G_{y}$, where $m \leq x \leq t-1$ and $i \leq y \leq n$ (i.e., no two jumping flipping links overlap).

Theorem 2.1 provides a simple way to determine all jumping flipping links from the coefficient vector $\left(b_{t-1}\right.$, $\left.b_{t-2}, \ldots, b_{i}, *\right)$. We only need to examine $\left(b_{t-1}, b_{t-2}, \ldots, b_{i}\right.$, *) from left to right, and find a jumping $m$-flipping link connecting $G_{m}$ and $G_{n}$, where $m-n>1$, if $b_{m}>b_{m-1}=b_{m-2}=$ $\ldots=b_{n+1}<b_{n}$. Moreover, the jumping flipping link connects the $(r+1)$ th $\mathrm{K}(d, m)$ of $G_{m}$ and the $(r+1)$ th $\mathrm{K}(d$, $n$ ) of $G_{n}$, where $r=b_{m-1}=b_{m-2}=\ldots=b_{n+1}$. Throughout this paper, we use $J_{u, v}^{w}$ to denote the jumping $u$-flipping link that connects the $w$ th $\mathrm{K}(d, u)$ of $G_{u}$ and the $w$ th $\mathrm{K}(d, v)$ of $G_{v}$, where $u-v>1$ (refer to Figure 3).

Let $D_{G_{m}}$ denote the diameter of $G_{m}$. Since $G_{m}$ contains $b_{m} \mathrm{~K}(d, m)$ 's that are completely connected, we have $D_{G_{m}}=2 D_{m}+1$ if $b_{m}>1$, and $D_{G_{m}}=D_{m}$ if $b_{m}=1$, where $D_{m}=2^{m}-1$ is the diameter of $\mathrm{K}(d, m)$ [2]. It has been shown in [2] that the distance between any two $m$ frontiers of $\mathrm{K}(d, m)$ defines $D_{m}$. Since the topology of $\operatorname{IK}(d, t)$ is much irregular than that of $\mathrm{K}(d, t)$, it is not surprising to see that computing the diameter of $\operatorname{IK}(d, t)$ is far more difficult than computing $D_{t}$. In fact, we are convinced that the diameter of $\operatorname{IK}(d, t)$ cannot be expressed with a formula. In subsequent sections, we use $I D_{t}$ to denote the diameter of $\operatorname{IK}(d, t)$, and $d(A, B)$ to denote the distance between any two nodes $A$ and $B$ in $\operatorname{IK}(d, t)$.

\section{An Upper Bound on $I D_{t}$}

An upper bound on $I D_{t}$ is proposed below.

Theorem 3.1. For $\operatorname{IK}(d, t)$ with coefficient vector $\left(b_{t-1}, b_{t-2}, \ldots, b_{i}, *\right)$, where $1 \leq i \leq t-1$, we have $I D_{t} \leq 2^{t}+2^{t-1}-$ $2^{i}-1$.

Proof. We prove this theorem by induction on $i$. Induction basis. When $i=t-1, b_{t-1} \geq 2$ and $I D_{t}=D_{G_{t-1}}=2^{t}-1$. This theorem holds.

Induction hypothesis. Assume this theorem holds for $i \geq m+1$, where $1 \leq m \leq t-2$. That is, $I D_{t} \leq 2^{t}+2^{t-1}-2^{m+1}-1$.

Induction step. We proceed with $i=m$. Suppose $U$ and $V$ are any two nodes of the $\operatorname{IK}(d, t)$. If they both reside in $G_{t-1}+G_{t-2}+\ldots+G_{m+1}$, we have by assumption $d(U, V) \leq$ $2^{t}+2^{t-1}-2^{m+1}-1<2^{t}+2^{t-1}-2^{m}-1$. If they both reside in $G_{m}$, we have $d(U, V) \leq 2 D_{m}+1=2^{m+1}-1<2^{i}+2^{t-1}-2^{m}-1$ because $m \leq t$ 2. So, for completeness, we assume $U$ and $V$ reside in $G_{l}$ and $G_{m}$, respectively, where $t-1 \geq l>m$, in the following discussion. Since there is at most one jumping flipping link incident on $G_{m}$ (known from Theorem 2.1), we consider the following two cases.
Case 1. There is one jumping flipping link connecting $G_{m}$ and $G_{k}$ for some $k, t-1 \geq k>m+1$.

Suppose $\left(w_{1}, w_{2}\right)$ is the jumping flipping link, where $w_{1} \in G_{k}$ and $w_{2} \in G_{m}$. We first assume $k \leq l$. Clearly $d(U, V) \leq d\left(U, w_{1}\right)+1+d\left(w_{2}, V\right)$. Since $d\left(w_{2}, V\right) \leq D_{G_{m}} \leq$ $2^{m+1}-1$ and $d\left(U, w_{1}\right) \leq 2^{t}+2^{t-1}-2^{k}-1$ by assumption, we have $d(U, V) \leq 2^{t}+2^{t-1}-2^{k}+2^{m+1}-1<2^{t}+2^{t-1}-2^{m}-1$ because $k \geq m+2$.

On the other hand, if $k>l$, we have $b_{k}>b_{k-1}=b_{k-2}=\ldots=$ $b_{l}=\ldots=b_{m+1}<b_{m}$ by Theorem 2.1. This implies that there is a path between $U$ and $V$ whose length is bound above by $\left(D_{l}+1\right)+\left(D_{l-1}+1\right)+\ldots+\left(D_{m+1}+1\right)+D_{G_{m}}=2^{l+1}-1$. So, $d(U$, $V) \leq 2^{l+1}-1<2^{t}+2^{t-1}-2^{m}-1$ because $l \leq t-2$ in this situation.

Case 2. No jumping flipping link is incident on $G_{m}$.

We first assume $b_{m+1}<b_{m}$. If $b_{l}=\min \left\{b_{l}, b_{l-1}, \ldots, b_{m}\right\}$, there is a path between $U$ and $V$ whose length is bound above by $\left(D_{l}+1\right)+\left(D_{l-1}+1\right)+\ldots+\left(D_{m+1}+1\right)+D_{G_{m}}=2^{l+1}-1$. So, $d(U, V) \leq 2^{l+1}-1<2^{t}+2^{t-1}-2^{m}-1$ because $l \leq t-1$ and $m \leq t-2$. Otherwise, let $b_{r}=\min \left\{b_{l}, b_{l-1}, \ldots, b_{m}\right\}$, where $m+1<r<l$ (if $r=m+1$, there is a jumping flipping link incident on $G_{m}$ ). By Theorem 2.1 there is exactly one jumping flipping link between $G_{x}$ and $G_{y}$, where $b_{x}>b_{x-1}=\ldots=b_{r}=$ $\ldots=b_{y+1}<b_{y}$. Let $\left(w_{1}, w_{2}\right)$ be the link and $w_{1} \in G_{x}, w_{2}$ $\in G_{y}$. Clearly $d(U, V) \leq d\left(U, w_{1}\right)+1+d\left(w_{2}, V\right)$. In addition, $d\left(U, w_{1}\right) \leq 2^{t}+2^{t-1}-2^{x}-1$ by assumption, and there is a path between $w_{2}$ and $V$ whose length is bound above by $\left(D_{G_{y}}+1\right)+\left(D_{y-1}+1\right)+\left(D_{y-2}+1\right)+\ldots+\left(D_{m+1}+1\right)+D_{G_{m}}=2^{y+1}+$ $2^{y-1}$. Hence, $d(U, V) \leq 2^{t}+2^{1-1}-2^{x}+2^{y+1}+2^{y}-1<2^{t}+2^{z-1}-2^{m}-1$ because $x>y+1$ and $y>m$.

On the other hand, if $b_{m+1} \geq b_{m}$, one (m+1)-flipping link connects each of the $\mathrm{K}(d, m)$ 's contained in $G_{m}$ to $G_{m+1}$. Since the distance between $U$ and any node of $G_{m+1}$ is not greater than $2^{t}+2^{t-1}-2^{m+1}-1$ (by assumption), we have $d(U, V) \leq 2^{t}+2^{t-1}-2^{m+1}-1+\left(D_{m}+1\right)=2^{t}+2^{t-1}-2^{m}-1$.

Q.E.D.

Note that the upper bound we proposed in Theorem 3.1 is tight for general $\operatorname{IK}(d, t)$. This can be seen as follows. Consider a particular $\operatorname{IK}(d, t)$ whose coefficient vector $\left(b_{t-1}, b_{t-2}, \ldots, b_{i}, *\right)$ satisfies $b_{t-1}>b_{t-2} \geq b_{t-3} \geq \ldots \geq b_{i}$. The distance between $\left(b_{t-1}-1\right)^{t}$ and $b_{t-1} b_{t-2} \ldots b_{i+1} 0(1)^{i}$ is equal to $\left(D_{G_{t-1}}+1\right)+\left(D_{t-2}+1\right)+\left(D_{t-3}+1\right)+\ldots+\left(D_{i+1}+1\right)+D_{i}=$ $2^{t}+2^{t-1}-2^{i}-1$, which matches the upper bound. However, for most instances of $\operatorname{IK}(d, t)$, their diameters are smaller than the upper bound. To calculate the actual values, an $O(t)$ time algorithm is proposed in the following section.

\section{An $O(t)$ Time Algorithm for Computing $I D_{t}$}


For $\operatorname{IK}(d, t)$ with coefficient vector $\left(b_{t-1}, b_{t-2}, \ldots, b_{i}\right.$, $*$ ), its diameter is simply $D_{G_{t-1}}$ if $i=t-1$. Thus we assume $1 \leq i \leq t-2$ throughout this section. Moreover, we let $U$ and $V$ denote one farthest pair of nodes in the $\operatorname{IK}(d, t)$, and assume that $U$ precedes $V$ lexicographically. Our algorithm aims to identify $U$ and $V$ and then compute their distance. Note that $d(U, V)=I D_{t}$.

It is impossible for both $U$ and $V$ to reside in $G_{t-2}+$ $G_{t-3}+\ldots+G_{i}$. The reason is explained as follows. If both $U$ and $V$ reside in $G_{t-2}+G_{t-3}+\ldots+G_{i}$, then by Theorem 3.1 we have $d(U, V) \leq 2^{t-1}+2^{t-2}-2^{i}-1$ because $G_{t-2}+G_{t-3}+\ldots$ $+G_{i}$ itself forms an $\operatorname{IK}(d, t-1)$ with coefficient vector $\left(b_{t-2}\right.$, $\left.b_{t-3}, \ldots, b_{i}, *\right)$. However, when $b_{t-1}>1$, the distance between any two $t$-frontiers is $2^{t}-1$; when $b_{t-1}=1$, the distance between a $t$-frontier in $G_{t-1}$ and a $(t-2)$-frontier (but not a $(t-1)$-frontier) in $G_{t-2}$ is at least $2^{t-1}+2^{t-2}-1$. They both are greater than $2^{t-1}+2^{t-2}-2^{i}-1$. Hence, $U$ or both $U$ and $V$ reside in $G_{t-1}$.

Note that there are $d-1(t-1)$-frontiers and one $t$ frontier for each $\mathrm{K}(d, t-1)$ within $G_{t-1}$. Observing the topology of $\operatorname{IK}(d, t)$, it is not difficult to see that for any $\mathrm{K}(d, t-1)$ within $G_{t-1}$, say $x \cdot \mathrm{K}(d, t-1)\left(0 \leq x \leq b_{t-1}-1\right)$, we have $d\left(v,(x)^{t}\right) \geq d(v, w)$ for any two nodes $v \notin x \cdot \mathrm{K}(d, t-1)$ and $w \in x \cdot \mathrm{K}(d, t-1)$. So, $U$ can be a $t$-frontier of some $\mathrm{K}(d, t-1)$ within $G_{t-1}$, and consequently $V$ is an $r$-frontier of some $\mathrm{K}(d, r)$ within $G_{r}, i \leq r \leq t-1$. Therefore, we have the following observation without further explanation.

Observation 4.1. For $\operatorname{IK}(d, t)$ with coefficient vector $\left(b_{t-1}, b_{t-2}, \ldots, b_{i}, *\right), U$ can be a $t$-frontier of some $\mathrm{K}(d, t-1)$ within $G_{t-1}$ and $V$ can be an $r$-frontier of some $\mathrm{K}(d, r)$ within $G_{r}$, where $i \leq r \leq t-1$.

When $b_{t-1}=1, I D_{t}$ can be computed as follows.

Theorem 4.1. For $\operatorname{IK}(d, t)$ with coefficient vector $\left(1, b_{t \cdot 2}, \ldots, b_{i}, *\right), I D_{t}$ can be computed in $O(t)$ time as follows.

(1) If $b_{j} \leq 1$ for all $i \leq j \leq t-1, I D_{t}=\sum_{\substack{i \leq j \leq t-1 \\ b_{j} \neq 0}} 2^{j}-1$.

(2) Otherwise, $I D_{t}=\sum_{\substack{k<j \leq t-1 \\ b_{j} \neq 0}} 2^{j}+2^{k+1}-1$, where $k=\max \{j \mid$ $i \leq j \leq t-1$ and $b \geq 2\}$.

Proof. According to Observation 4.1, we can let $U=(0)^{t}$. If $b_{j} \leq 1$ for all $i \leq j \leq t-1$, at most one $\mathrm{K}(d, j)$ is contained in $G_{j}$ and all of them are linearly connected. Hence, $V=b_{t-1} b_{t-2} \ldots b_{i+1} O(1)^{i}$ is one farthest node from $U$, and $d(U, V)$ is computed as the summation of $\left(D_{j}+1\right)$ for all $j \in\left\{m \mid b_{m} \neq 0\right.$ and $\left.i<m \leq t-1\right\}$ plus $D_{i}$, which is simplified to $\sum_{\substack{i \leq j \leq t-1 \\ b_{j} \neq 0}} 2^{j}-1$. Note that the selection of $V$ is not unique.

On the other hand, since $b_{k} \geq 2$, we have $D_{G_{k}}=2^{k+1}-1$.

Let $w$ be the $(k+1)$-frontier of $b_{t-1} b_{t-2} \ldots b_{k+1} 0 \cdot \mathrm{K}(d, k)$ (within $G_{k}$ ). It is clear that $V$ must reside in $G_{k}+G_{k-1}+\ldots$ $+G_{i}$ and $d(U, V)=d(U, w)+d(w, V)$. Assume $v$ belongs to $G_{k-1}+\ldots+G_{i}$, say $v \in G_{r}, i \leq r \leq k-1$. It is not difficult to see that $d(w, v)$ is not greater than the summation of $\left(D_{j}+1\right)$ for all $j \in\left\{m \mid b_{m} \neq 0\right.$ and $\left.r<m \leq k\right\}$ plus $D_{G_{r}}$, which is at most $2^{k+1}-1$. However, $w$ and $b_{t-1} b_{t-2} \ldots b_{k+1} 1(1)^{k}$ are one farthest pair of nodes in $G_{k}$, and their distance is $D_{G_{k}}=2^{k+1}-1 \geq d(w, v)$. This implies $V$ can be $b_{t-1} b_{t-2} \ldots$ $b_{k+1} 1(1)^{k}$. The value $d(U, V)$ is computed as the summation of $\left(D_{j}+1\right)$ for all $j \in\left\{m \mid b_{m} \neq 0\right.$ and $\left.k<m \leq t-1\right\}$ plus $D_{G_{k}}$, which is simplified to $\sum_{\substack{k<j \leq t-1 \\ b_{j} \neq 0}} 2^{j}+2^{k+1}-1$.

Q.E.D.

In the rest of this section, we concentrate our attention on $b_{t-1} \geq 2$. We first show that $\operatorname{IK}(d, t)$ with coefficient vector $\left(b_{t-1}, b_{t-2}, \ldots, b_{i}, *\right)$ can be pruned appropriately so that the pruned structure has the same diameter. We define $\bar{b}_{j}=\min \left\{b_{t-1}, b_{j}\right\}$ for all $i \leq j \leq t-1$. The pruned structure is obtained by removing from $\operatorname{IK}(d, t)$ $b_{t-1} b_{t-2} \ldots b_{j+1} \bar{b}_{j} \cdot \mathrm{K}(d, j), b_{t-1} b_{t-2} \ldots b_{j+1}\left(\bar{b}_{j}+1\right) \cdot \mathrm{K}(d, j), \ldots$, $b_{t-1} b_{t-2} \ldots b_{j+1}\left(b_{j}-1\right) \cdot \mathrm{K}(d, j)$ if $\bar{b}_{j}<b_{j}$ for all $i \leq j<t-1$. For example, consider $\operatorname{IK}(d, 6)$ with coefficient vector $(2,3$, $4,1, *)$. We have $\bar{b}_{5}=2, \bar{b}_{4}=2, \bar{b}_{3}=2, \bar{b}_{2}=1$, and the pruned structure contains $0 \cdot \mathrm{K}(d, 5), 1 \cdot \mathrm{K}(d, 5), 20 \cdot \mathrm{K}(d, 4)$, $21 \cdot \mathrm{K}(d, 4), 230 \cdot \mathrm{K}(d, 3), 231 \cdot \mathrm{K}(d, 3)$, and $2340 \cdot \mathrm{K}(d, 2)$. The pruned structure is actually a subgraph of the $\operatorname{IK}(d, t)$. Let $\overline{I D}_{t}$ denote the diameter of the pruned structure of $\mathrm{IK}(d, t)$. As proven in [21], we have $\overline{I D}_{t}=I D_{l}$.

Theorem 4.2.[21] For $\operatorname{IK}(d, t)$ with coefficient vector $\left(b_{t-1}, b_{t-2}, \ldots, b_{i,} *\right.$, we have $\overline{I D}_{t}=I D_{t}$ for $b_{t-1} \geq 2$.

See Figure 4(a) which is the pruned structure of $\operatorname{IK}(6$, 10) with coefficient vector $(4,3,5,1,1,5,2,5,1, *)$ (illustrated in Figure 3(b)). In Figure 4(a), we have defined $\bar{G}_{x}(i \leq x \leq t-1)$ to be the subgraph of $\operatorname{IK}(d, t)$ with coefficient vector $\left(b_{t-1}, b_{t-2}, \ldots, b_{i}, *\right)$ induced by the nodes of $\left.b_{t-1} b_{t-2} \ldots b_{x+1} 0 \cdot \mathrm{K}(d, x), b_{t-1} b_{t-2} \ldots b_{x+1} 1 \cdot \mathrm{K}(d, x)\right)$, $\ldots$, and $b_{t-1} b_{t-2} \ldots b_{x+1}\left(\bar{b}_{x}-1\right) \cdot \mathrm{K}(d, x)$.

Now is the time to present our algorithm for computing the diameter of $\operatorname{IK}(d, t)$ with coefficient vector $\left(b_{t-1}, b_{t-2}, \ldots, b_{i}, *\right)$, where $b_{t-1} \geq 2$. The algorithm consists 


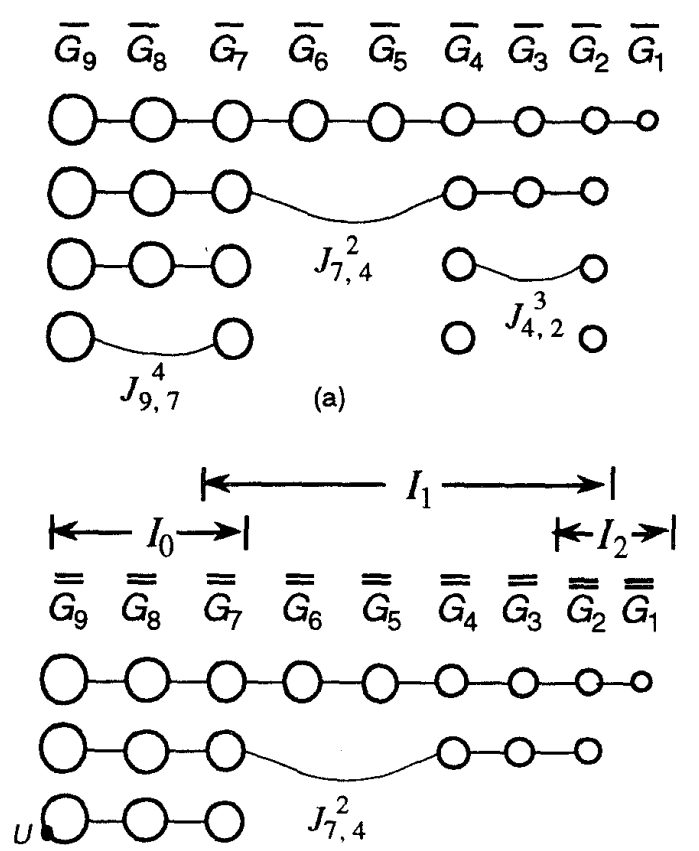

(b)

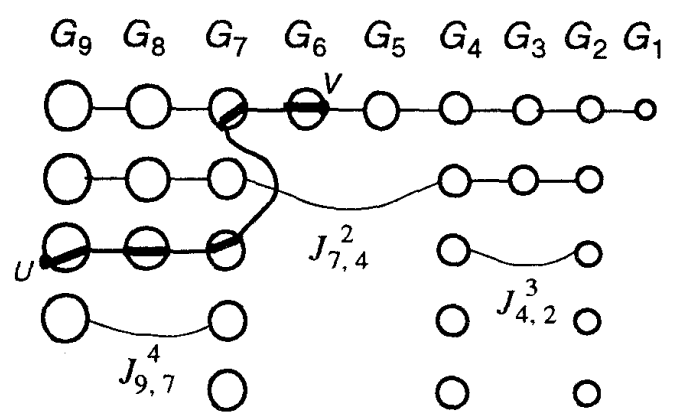

(c)

Figure 4. An example.

of five stages, and it can identify $U$ and $V$ and compute $d(U, V)$ in $O(t)$ time from $\bar{b}_{t-1}, \bar{b}_{t-2}, \ldots, \bar{b}_{i}$. In the following the five stages are described one after another.

Stage 1. Scan $\bar{b}_{t+1}, \bar{b}_{t-2}, \ldots, \bar{b}_{i}$ from left to right and determine in sequence $J_{y_{1}, z_{1}}^{x_{1}}, J_{y_{2}, z_{2}}^{x_{2}}, \ldots, J_{y_{n}, z_{n}}^{x_{n}}$ so that $x_{1}>x_{2}>\ldots>x_{n}$. That is, $J_{y_{1}, z_{1}}^{x_{1}}$ is the first one encountered in the scanning, each $J_{u, v}^{w}$ between $J_{y_{j}, z_{j}}^{x_{j}}$ and $J_{y_{j+1}, z_{j+1}}^{x_{j+1}}$, where $1 \leq j<n$, has $w \geq x_{j}$, and each $J_{u, v}^{w}$ after $J_{y_{n}, z_{n}}^{x_{n}}$ has $w \geq x_{n}$. Then store $x_{1}, x_{2}, \ldots, x_{n}$ in a linked list $L$. If no feasible jumping flipping link exists, then $L$ is empty.

For the example of Figure $4(\mathrm{a}), x_{1}=4, x_{2}=2$, and $L=(4,2)$. By the aid of Theorem 2.1 Stage 1 can be completed in $O(t)$ time.

Stage 2. Determine $\alpha=\max \left\{r \mid r \notin L\right.$ and $\left.1 \leq r \leq \bar{b}_{t-1}\right\}$. If $\alpha=1$ or no feasible $\alpha$ exists, $U$ and $V$ are determined as $(0)^{t}$ and $(1)^{t}$, respectively, and $d(U, V)$ is computed as $2^{t}$ 1. If $\alpha \geq 2, U$ is determined as the $t$-frontier of the $\alpha$ th $\mathrm{K}(d$, $t-1)$. For the latter case, remove from $\boldsymbol{L}$ those values that are greater than $\alpha$.

If $\alpha=1$ or no feasible $\alpha$ exists, the execution will terminate. If $\alpha \geq 2, L$ may become empty or contain $e_{1}>$ $e_{2}>\ldots>e_{k}$, and the execution then proceeds to the next stage. For the example above, $\alpha=3, U$ is determined as $(2)^{10}$ and $L$ contains $e_{1}=2$ only. Clearly Stage 2 can be completed in $O(t)$ time.

Stage 3. Determine $m_{0}$ as the least index $r$ such that $\bar{b}_{s} \geq \alpha$ for $t-1 \geq s \geq r$. If $L$ contains $e_{1}>e_{2}>\ldots>e_{k}$, determine $m_{1}, m_{2}, \ldots, m_{k}$ sequentially as follows: $m_{1}=\min \{r \mid$ $\bar{b}_{r} \geq e_{1}$ and $\bar{b}_{s} \geq e_{1}-1$ for $\left.m_{0}>s>r\right\}, m_{2}=\min \left\{r \mid \bar{b}_{r} \geq e_{2}\right.$ and $\bar{b}_{s} \geq e_{2}-1$ for $\left.m_{1}>s>r\right\}, \ldots$, and $m_{k}=\min \left\{r \mid \bar{b}_{r} \geq e_{k}\right.$ and $\bar{b}_{s} \geq e_{k}-1$ for $\left.m_{k-1}>s>r\right\}$.

Note that $t-1 \geq m_{0}>m_{1}>\ldots>m_{k-1}>m_{k} \geq i$. In the example above, since $\alpha=3, k=1$, and $e_{1}=2$, we have $m_{0}=7$, $m_{1}=2$. The values $m_{0}, m_{1}, \ldots, m_{k}$ can be determined in $O(t)$ time by scanning $\bar{b}_{t-1}, \bar{b}_{t-2}, \ldots, \bar{b}_{i}$ from left to right.

Stage 4. The pruned structure is further pruned. The second pruned structure is obtained by removing from the first pruned structure $b_{t-1} b_{t-2} \ldots b_{j+1} \overline{\bar{b}}_{j} \cdot \mathrm{K}(d, j), b_{t-1} b_{t-2} \ldots$ $b_{j+1}\left(\overline{\bar{b}}_{j}+1\right) \cdot \mathrm{K}(d, j), \ldots, b_{t-1} b_{t-2} \ldots b_{j+1}\left(\bar{b}_{j}-1\right) \cdot \mathrm{K}(d, j)$ if $\overline{\bar{b}}_{j}<$ $\bar{b}_{j}$, where $\overline{\bar{b}}_{j}=\alpha$ for all $t-1 \geq j \geq m_{0} ; \overline{\bar{b}}_{j}=\min \left\{e_{r}, \bar{b}_{j}\right\}$ if $e_{r}>1$ and $\bar{b}_{F}=\bar{b}_{j}$ if $e_{r}=1$ for all $m_{r} \leq j<m_{r-1}$ and $1 \leq r \leq k ; \overline{\bar{b}}_{j}=\bar{b}_{j}$ for all $i \leq j<m_{k}$ if $m_{k}>i$.

In the example, we have $\overline{\bar{b}}_{9}=3, \overline{\bar{b}}_{8}=3, \overline{\bar{b}}_{7}=3, \overline{\bar{b}}_{6}=1$, $\overline{\bar{b}}_{5}=1, \overline{\bar{b}}_{4}=2, \overline{\bar{b}}_{3}=2, \overline{\bar{b}}_{2}=2$, and $\overline{\bar{b}}_{1}=1$ Refer to Figure 4(b) where we define $\overline{\bar{G}}_{x}(i \leq x \leq t-1)$ to be the subgraph of $\mathrm{IK}(d, t)$ with coefficient vector $\left(b_{t-1}, b_{t-2}, \ldots, b_{i}, *\right)$ induced by the nodes of $b_{t-1} b_{t-2} \ldots b_{x+1} 0 \cdot \mathrm{K}(d, x), b_{t-1} b_{t}$. $2 \ldots b_{x+1} 1 \cdot \mathrm{K}(d, x), \ldots$, and $\left.b_{t-1} b_{t-2} \ldots b_{x+1}\left(\overline{\bar{b}}_{x}-1\right) \cdot \mathrm{K}(d, x)\right)$. Clearly, it takes $O(t)$ time to determine $\bar{b}_{t-1}, \bar{b}_{t-2}, \ldots, \overline{\bar{b}}_{i}$. 
For the convenience of explanation, we define intervals $I_{0}, I_{1}, \ldots$ as follows. If $L$ is empty, define $I_{0}=\left[m_{0}, t-1\right]$ and $I_{1}=\left[i, m_{0}\right]$, where $I_{1}$ exists only when $i<m_{0}$. If $\boldsymbol{L}$ is not empty, define $I_{0}=\left[m_{0}, t-1\right], I_{r}=\left[m_{r}\right.$, $\left.m_{r-1}\right]$ for $1 \leq r \leq k$, and $I_{k+1}=\left[i, m_{k}\right]$, where $I_{k+1}$ exists only when $i<m_{k}$ (refer to Figure 4(b)). We regard $i$ as $m_{k+1}$ if $I_{k+1}$ exists (i.e., $I_{k+1}=\left[m_{k+1}, m_{k}\right]$ ). For each interval $I_{j}=[g$, $h$ ], we define $S_{j}$ to be the set of jumping flipping links that are contained in $\overline{\bar{G}}_{h}+\overline{\bar{G}}_{h-1}+\ldots+\overline{\bar{G}}_{g}$. Note that according to the definitions of $m_{0}, m_{1}, \ldots, m_{k}$, when $L$ is not empty, we have $\left|S_{0}\right|=0,\left|S_{r}\right| \geq 1$ for $1 \leq r \leq k$, and $\left|S_{k+1}\right|=0$ provided $I_{k+1}$ exists. When $L$ is empty, we have $\left|S_{0}\right|=0$ and $\mid S_{1} \models 0$ provided $I_{1}$ exists.

Stage 5. If there exists only one interval $I_{0}=\left[m_{0}, t-1\right]$ (i.e., $\left.m_{0}=i\right), V$ is determined as the $t$-frontier of the ( $\alpha$ 1)th $\mathrm{K}(d, t-1)$ within $G_{t-1}$ and $d(U, V)$ is computed as $2^{t}-1$. Otherwise, suppose there are $l>1$ intervals, $I_{0}, I_{1}, \ldots, I_{l-1}$. Then $V$ and $d(U, V)$ can be determined according to the following procedure.

4.1. $d i s \leftarrow 2^{i}-2^{m_{0}}$.

4.2. For each interval, say $I_{j}=[g, h]$, in the sequence of $I_{1}, I_{2}, \ldots, I_{l-2}$, if $S_{j}$ contains only one jumping flipping link, say $J_{y, z}^{x}$, and $y-z=2$, then dis $\leftarrow$ dis $+2^{h+1}-2^{y-1}-2^{g}$. Otherwise, letting $J_{y, z}^{x}$ be the one with maximum $y$ in $S_{j}, V \leftarrow b_{t-1} b_{t}$. ${ }_{2} \ldots b_{y} 0(1)^{y-1}, d(U, V) \leftarrow d i s+2^{h+1}-2^{y-1}-1$, and the execution terminates.

4.3. Suppose $I_{l-1}=[i, c]$. If $\left|S_{l-1}\right|=0$, then $V \leftarrow b_{l-1} b_{l-2}$ $\ldots b_{i+1} 0(1)^{i}$ and $d(U, V) \leftarrow d i s+2^{c+1}-2^{i}-1$.

Otherwise, letting $J_{y, z}^{x}$ be the one with maximum $y$ in $S_{l-1}$, two cases are considered as follows.

Case 1. $x>1$, $V \leftarrow b_{t-1} b_{t-2} \ldots b_{y} 0(1)^{y-1}$ and $d(U, V) \leftarrow d i s+2^{c+1}$. $2^{y-1}-1$.

Case 2. $x=1$.

$V \leftarrow b_{t-1} b_{t-2} \ldots b_{k+1} 1(1)^{k}$ and $d(U, V) \leftarrow$ dis+ $\sum_{\substack{k<j \leq c \\ b_{j} \neq 0}} 2^{j}+2^{k+1}-1$, where $k=\max \left\{s \mid \overline{\bar{b}}_{s} \geq 2\right.$ and $i \leq$ $\bar{b}_{j} \neq 0$

$s \leq z\}$. If no feasible $k$ exists, then $V \leftarrow b_{t-1} b_{t-2} \ldots$ $b_{i+1} O(1)^{i}$ and $d(U, V) \leftarrow \operatorname{dis}+\sum_{\substack{i \leq j \leq c \\ \bar{b}_{j} \neq 0}} 2^{j}-1$.

For the example above, there are three intervals: $I_{0}=[7,9], I_{1}=[2,7]$, and $I_{2}=[1,2]$. Initially, we have dis $=2^{10}-2^{7}$ which represents the summation of $D_{9}+1+D_{8}+$ $1+D_{7}+1$. Next, since $S_{1}=\left\{J_{7,4}^{2}\right\}$, the execution terminates with $V=4350(1)^{6}$ and $d(U, V)=\left(2^{10}-2^{7}\right)+\left(2^{8}-2^{6}-1\right)$, where $2^{8}-2^{6}-1$ represents the summation of $D_{7}+1+D_{6}$. So, for $\operatorname{IK}(6,10)$ with coefficient vector $(4,3,5,1,1,5,2,5,1$, $*), U=(2)^{10}$ and $V=4350(1)^{6}$ are one farthest pair of nodes and the diameter is $d(U, V)=2^{10}+2^{6}-1$ which represents the summation of $D_{9}+1+D_{8}+1+D_{7}+1+D_{7}+1+D_{6}$ (refer to Figure $4(\mathrm{c}))$. Stage 5 can be completed in $O(t)$ time.

Lemma 4.1.[21] In Stage 2, if $\alpha=1$ or no feasible $\alpha$ exists, then $d(U, V)=\overline{I D}_{l}=2^{t}-1$. Otherwise, $(\alpha-1)^{t}$ is a feasible node for $U$.

Lemma 4.2.[21] In Stage 4, the diameter of $\overline{\bar{G}}_{t-1}+\overline{\bar{G}}_{t-2}+\ldots+\overline{\bar{G}}_{i}$ is equal to $\overline{D D}_{t}$.

Lemma 4.3.[21] Node $V$ determined in Stage 5 satisfies $d(U, V)=\overline{I D}_{t}$.

Recall that $I D_{t}=D_{G_{t-1}}$ for $\operatorname{IK}(d, t)$ with coefficient vector $\left(b_{t-1}, *\right)$. Combining Theorems 4.1, 4.2, Lemmas $4.1,4.2$, and 4.3 , we reach the main conclusion of this section as follows.

Theorem 4.3. For $\operatorname{IK}(d, t)$ with coefficient vector $\left(b_{t-1}, b_{t-2}, \ldots, b_{i}, *\right), 1 \leq i \leq t-1, I D_{t}$ and a feasible pair of nodes $U$ and $V$ such that $d(U, V)=I D_{t}$ can be determined in $O(t)$ time.

\section{Concluding Remarks}

Computing the diameter for an incomplete network is far more difficult than that for a complete network. The reason is that complete networks of different sizes preserve great topological similarity, whereas incomplete networks may have a significant difference in their topologies. For example, $\mathrm{K}(d, t)$ looks very similar to $\mathrm{K}(d, t-1)$, whereas two $\operatorname{IK}(d, t)$ 's with different coefficient vectors may look very unlike in their topologies. The diameter is still unknown for the incomplete star networks [14, 18] and the incomplete rotator graphs [16], although it has been well solved for the star networks [1] and the rotator graphs [3].

In this paper, by the aid of the multistage graph representation, we first suggested a tight upper bound on the diameters of the incomplete WK-recursive networks. Then, we proposed a linear-time algorithm for computing the diameters. The algorithm adopts the prune-and-search technique [15] which has proven an efficient method for solving problems from operations research and computational geometry. In the algorithm $G_{t-1}+G_{t-2}+\ldots$ $+G_{i}$ is first pruned to form a reduced structure $\bar{G}_{t-1}+\bar{G}_{t-2}+$ $\ldots+\bar{G}_{i}$, which is further pruned into $\overline{\bar{G}}_{t-1}+\overline{\bar{G}}_{t-2}+\ldots$ 
$+\overline{\bar{G}}_{i}$. All these three structures, although different in their sizes, have the same diameter. Thus, the diameter of $\overline{\bar{G}}_{t-1}$ $+\overline{\bar{G}}_{t-2}+\ldots+\overline{\bar{G}}_{i}$ is computed as the diameter of $G_{t-1}+G_{t-2}+$ $\ldots+G_{i}$.

More topological properties, including the connectivity and hamiltonicity, can be found in [21]. Besides, a shortest-path routing algorithm and a broadcasting algorithm have been presented in [22] and [23], respectively. The authors are now trying to solve more problems on the incomplete WK-recursive networks.

\section{References}

[1] S. B. Akers and B. Krishnamurthy, "A grouptheoretic model for symmetry interconnection networks," IEEE Trans. on Computers, vol. 38, no. 4, pp. 555-566, 1989.

[2] G. H. Chen and D. R. Duh, "Topological properties, communication, and computation on WK-recursive networks," Networks, vol. 24 , no. 6 , pp. 303-317, 1994.

[3] P. Corbett, "Rotator graphs: an efficient topology for point-to-point multiprocessor networks," IEEE Trans. on Parallel and Distributed Systems, vol. 3, no. 5, pp. 622-626, 1992.

[4] K. Day and A. Tripathi, "Arrangement graphs: a class of generalized star graphs," Information Processing Letters, vol. 42, no. 5, pp. 235-241, 1992.

[5] D. R. Duh and G. H. Chen, "Topological properties of WK-recursive networks," $J$. of Parallel and Distributed Computing, vol. 23, no. 3, pp. 468474, 1994.

[6] D. R. Duh and G. H. Chen, "On the Rabin number problem," submitted.

[7] R. Fernandes, "Recursive interconnection networks for multicomputer networks," in Proc. of the Int. Conf. on Parallel Processing, pp. 76-79, 1992.

[8] R. Fernandes and A. Kanevsky, "Hierarchical WKrecursive topologies for multicomputer systems," in Proc. of the Int. Conf. on Parallel Processing, vol. 1, pp. 315-318, 1993.

[9] R. Fernandes, D. K. Griesen, and A. Kanevsky, "Efficient routing and broadcasting in recursive interconnection networks," in Proc. of the Int. Conf. on Parallel Processing, 1994, pp. 51-58.

[10] R. Fernandes and A. Kanevsky, "Substructure allocation in recursive interconnection networks," in Proc. of Inte Conf. on Parallel Processing, vol. 1, 1993, pp. 319-322.

[11] R. Fernandes, D. K. Friesen, and A. Kanevsky, "Embedding rings in recursive networks," in Proc. of Int. Symp. on Parallel and Distributed Processing, Oct. 1994, pp. 273-280.
[12] A. Iazzetta, C. Sanges, and U. Scafuri, "Special multicast wormhole routing for WK-networks," manuscript.

[13] H. P. Katseff, "Incomplete hypercubes," IEEE Trans. on Computers, vol. C-37, no. 5, pp. 604608, 1988.

[14] S. Latifi and N. Bagherzadeh, "Incomplete star: an incrementally scalable network based on the star graph," IEEE Trans. on Parallel and Distributed Systems, vol. 5, no. 1, pp. 97-102, 1994.

[15] D. T, Lee and F. P. Preparata, "Computational geometry - a survey," IEEE Trans. on Computers, vol. C-33, pp. 1072-1101, Dec. 1984.

[16] S. Ponnuswamy and V. Chaudhary, "Embedding of cycles in rotator and incomplete rotator graphs," in Proc. of Int. Symp. on Parallel and Distributed Processing, Oct. 1994, pp. 603-610.

[17] F. P. Preparata and J. Vuillemin, "The cubeconnected cycles: a versatile network for parallel computation," Communications of the ACM, vol. 24, no. 5, pp. 300-309, 1981.

[18] C. P. Ravikumar, A. Kuchlous, and G. Manimaran, "Incomplete star graph: an economical fault-tolerant interconnection network," in Proc. of Int. Conf. on Parallel Processing, vol. 1, 1993, pp. 83-90.

[19] M. R. Samatham and D. K. Pradhan, "The de Bruijn multiprocessor networks: a versatile parallel processing and sorting networks for VLSI," IEEE Trans. on Computers, vol. 38, pp. 567-581, 1989.

[20] H. S. Stone, "Parallel processing with the perfect shuffle," IEEE Trans. on Computers, vol. 20, no. 2, pp. 153-161, 1971.

[21] M. Y. Su, G. H. Chen, and D. R. Duh, "Topological properties of incomplete WK-recursive networks," Tech. Rep. 95-06, Dept. of Comput. Sci. and Info. Engg., National Taiwan Univ., Taipei, Taiwan, March 1995.

[22] M. Y. Su, G. H. Chen, and D. R. Duh, "A shortest-path routing algorithm for the incomplete WK-recursive networks," Tech. Rep. 95-07, Dept. of Comput. Sci. and Info. Engg., National Taiwan Univ., Taipei, Taiwan, July 1995.

[23] M. Y. Su, G. H. Chen, and D. R. Duh, "A broadcasting algorithm for the incomplete WKrecursive networks," in Proc. of Inter. Symposium on Parallel Architecture, Algorithm, and Networks (ISPAN), 1996, to appear.

[24] G. D. Vecchia and C. Sanges, "A recursively scalable network VLSI implementation," Future Generation Computer Systems, vol. 4, no. 3, pp. 235-243, 1988.

[25] G. D. Vecchia and C. Sanges, "An optimized broadcasting technique for WK-recursive topologies, "Future Generation Computer Systems, vol. 4, no. 3, pp. 353-357, 1989/90. 